\title{
Buckling and enforced stretching of bio-filaments
}

\author{
Bin $\mathrm{Hu}^{1}$, V.B. Shenoy ${ }^{2}$ and Yuan $\operatorname{Lin}^{1 *}$ \\ ${ }^{1}$ Department of Mechanical Engineering, The University of Hong Kong, Hong Kong SAR, China \\ ${ }^{2}$ School of Engineering, Brown University, Providence, RI, USA \\ *Corresponding author: ylin@hku.hk
}

\begin{abstract}
We examine the force needed to extend/compress a bio-filament, a key issue in the study of cytoskeleton mechanics and polymer physics, by considering both the associated stretching and bending deformations. Specifically, closed form relationships are derived to predict the buckling of stiff filaments such as F-actin and microtubules. Our results clearly demonstrate that the maximum force a 2D filament can sustain is higher than the Euler buckling load whereas the force in a 3D filament is always below it, and hence clarify some of the ambiguities in the literature. In addition, analytical expression is also obtained to describe how the extensional force increases when a flexible molecule, like DNA, is stretched close to its contour length, which has been shown to fit to a variety of experimental data very well. Our theory provides important corrections/improvements to several well-known existing models.
\end{abstract}

Keywords: Buckling, Bio-filament, Thermal fluctuations, Force-extension relationship

\section{Introduction}

Bio-filaments, such as F-actin and microtubule, are weak chains assembled from discrete protein monomers which can be further bundled or crosslinked to form the cytoskeleton or subcellular structures like the stress fibers interconnecting different adhesion sites. It is well documented that the force generation, presumably by polymerization or due to myosin motors, and load bearing capabilities of these filaments are essential to processes like cell migration (Pollard and Borisy, 
2003) and cytokinesis (Eggert et al., 2006). As such, great attention has been devoted to examine how various bio-filaments resist deformation as well as its implications to their physiological functions (Fletcher and Mullins, 2010; MacKintosh, 2006). Specifically, it has been found that thermal excitation alone is significant enough to perturb the shape of these weak rod-like structures (Gittes et al., 1993; Isambert et al., 1995). Buckling of a single filament immersed in a thermal reservoir was considered by different researchers (Baczynski et al., 2007; Lee et al., 2007; Gholami et al., 2006; Li, 2008; Odijk, 1998; Blundell and Terentjev, 2009) and its relevance in interpreting the mechanical behaviour of a filament network has also been examined (Liu et al., 2008; Chaudhuri et al., 2007).

In contrast to stiff filaments like short F-actin or microtubules, many long chain molecules such as DNA can only support tensile load. The mechanics involved in stretching a flexible molecule is best summarized by the so-called force-extension relationship, a focal point in the study of polymer physics. Historically, such relationship was first derived from the freely joint chain (FJC) picture, where the tensile force, $F$, needed to stretch a molecule close to its contour length, $L$, is predicted to diverge as $F \propto 1 / \varepsilon$. Here $\varepsilon=\left(L-L^{\prime}\right) / L$ with $L^{\prime}$ being the actual end-to-end distance of the chain under stretch. However, experiments conducted on DNAs (Smith et al., 1992) suggested that this force is actually proportional to $1 / \varepsilon^{2}$, which lead to the theory by Marko and Siggia (1995) based on the worm-like-chain (WLC) description where the bending of the molecule was taken into account. Different force-extension relationships listed above, as well as others not mentioned here, provide the basis for explaining and predicting the mechanical response of various polymer materials, like rubber (Arruda and Boyce, 1993) and biological gels (Gardel et al., 2004; Storm et al., 2005), as well as analyzing the enforced unfolding of proteins (Su and Purohit, 2009). One thing needs to be pointed out is that the filament is assumed to be inextensible in both the FJC and WLC formulations. However, numerous observations (Wang et al., 1997; Smith et al., 1996; Liu and Pollack, 2002) have convincingly demonstrated that molecules like DNA or long F-actin can actually be stretched beyond their contour lengths, suggesting that a more accurate model should take the axial deformation of filament into account. Indeed, an early attempt to address this issue has been made by Odijk (1995). 
Despite all these efforts, several fundamental questions remain unsettled. For example, some studies (Baczynski et al., 2007; Lee et al., 2007) have suggested that, in 2D, thermal excitation actually strengthens a filament under compression, that is, the maximum force the filament can sustain is higher than the Euler buckling load. On the other hand, it has also been reported that filaments should be weakened by temperature effect in 3D (Odijk, 1998; Blundell and Terentjev, 2009). However, a unified theory capable of explaining whether and why, if indeed, filaments behave differently in 2D and 3D is still lacking. In addition, although great attention has been paid to how the force increases when a filament is stretched close to its contour length, a rigorous analysis is not available. To address these issues, we revisit the problem of a filament under compression/stretching by considering both bending and stretching of the chain. We show that indeed the buckling behaviour of a filament in 2D is different from that in 3D. In addition, we further demonstrate that some existing force-extension relationships, such as that by Marko and Siggia (1995) or by Odijk (1995), are correct from the point of view of scaling but tend to considerably over-predict the force.

\section{Theory}

Consider a simply supported filament, with contour length $L$ and both ends located on the $x$ axis, is compressed through a distance $\delta=L-L^{\prime}$ as shown in Fig. 1. Notice that, here we consider the problem in a displacement controlled setup, that is, the compression distance $\delta$ is prescribed, so that the nominal compressive strain is

$$
\varepsilon=\frac{\delta}{L}
$$

Assume that the stretching and bending rigidity of the filament are $\kappa_{s}$ and $\kappa_{b}$ respectively. We proceed by normalizing the physical parameters as $\tilde{\kappa}_{s}=\frac{\kappa_{s} L}{k_{B} T}, \tilde{\kappa}_{b}=\frac{\kappa_{b}}{k_{B} T L}, \widetilde{U}=\frac{U}{k_{B} T}, \tilde{F}=\frac{F L}{k_{B} T}$, $\widetilde{L^{\prime}}=\frac{L^{\prime}}{L}=1-\varepsilon$, where $k_{B} T$ is the thermal energy, $F$ is the force applied at both ends of the filament and $U$ is the elastic energy stored. We use $u(x)$ and $v(x)$ to denote filament deflections (defined in the deformed configuration and normalized by $L$ ) in the $y$ and $z$ directions, 
respectively. If we restrict our attention to cases where the deflections are small, then the total elastic energy induced by the deformation can be calculated as

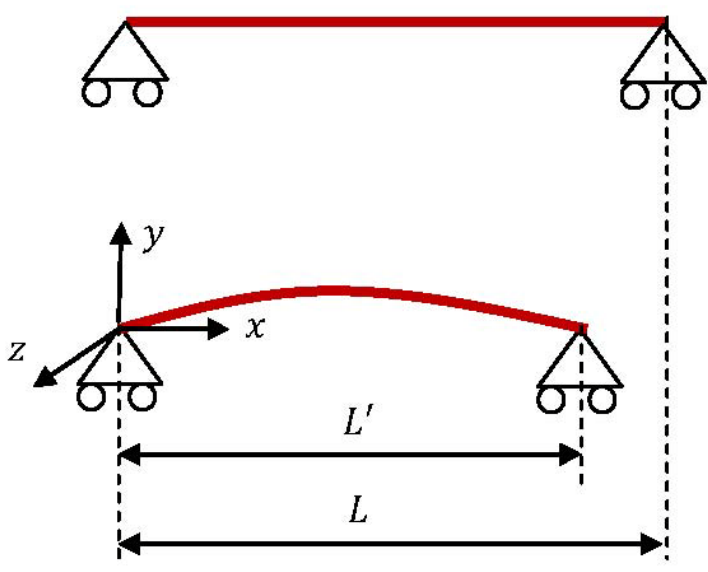

Figure 1. Schematic plot of a filament under compression.

$$
\widetilde{U}=\frac{1}{2} \tilde{\kappa}_{s} \varepsilon_{r}^{2}+\frac{1}{2} \tilde{\kappa}_{b} \int_{0}^{\widetilde{L \prime}}\left[\left(\frac{\partial^{2} u}{\partial x^{2}}\right)^{2}+\left(\frac{\partial^{2} v}{\partial x^{2}}\right)^{2}\right] d x
$$

where $\varepsilon_{r}=1-\int_{0}^{\widetilde{L^{\prime}}} \sqrt{1+\left[u^{\prime}(x)\right]^{2}+\left[v^{\prime}(x)\right]^{2}} d x$ is the axial strain in the filament and, like other length variables, $x$ has been normalized by $L$ here. Notice that, for a simply supported filament, the deflection profile can be expressed as

$$
u(x)=\sum_{n=1}^{\infty} a_{n} \sin \left(\frac{n \pi x}{\widetilde{L}^{\prime}}\right), \quad v(x)=\sum_{n=1}^{\infty} b_{n} \sin \left(\frac{n \pi x}{\widetilde{L}^{\prime}}\right) .
$$

For small $\varepsilon$, the axial strain can be found as $\varepsilon_{r} \approx 1-\int_{0}^{\widetilde{L \prime}}\left[1+\left[u^{\prime}(x)\right]^{2} / 2+\left[v^{\prime}(x)\right]^{2} / 2\right] d x \approx$ $\varepsilon-\frac{\pi^{2}}{4} \sum_{n} n^{2}\left(a_{n}^{2}+b_{n}^{2}\right)$, where higher order terms in $\varepsilon$ (or equivalently in $a_{n}^{2}$ and $b_{n}^{2}$ ) have been neglected. As such, the total energy stored is

$$
\widetilde{U}=\frac{1}{2} \tilde{\kappa}_{s}\left[\varepsilon-\frac{\pi^{2}}{4} \sum_{n} n^{2}\left(a_{n}^{2}+b_{n}^{2}\right)\right]^{2}+\frac{1}{4} \tilde{\kappa}_{b} n^{4} \pi^{4} \sum_{n}\left(a_{n}^{2}+b_{n}^{2}\right) .
$$


In addition to the elastic energy, we also need to take into account the entropy of the system when such filament is immersed in a thermal bath. A standard way to achieve this is to analyze the so-called free energy which, within the framework of classical statistical mechanics, can be calculated as $\widetilde{\mathrm{H}}=-\ln \mathrm{Z}$, with $\mathrm{Z}=\int_{-\infty}^{\infty} \ldots \int_{-\infty}^{\infty} \mathrm{e}^{-\widetilde{\mathrm{U}}} \mathrm{da}_{1} \ldots \mathrm{da}_{\mathrm{n}} \mathrm{db}_{1} \ldots \mathrm{db}_{\mathrm{n}}$ being the partition function of the system (Dill and Bromberg, 2002). Once $\widetilde{\mathrm{H}}$ is known, the force needed to be applied at both ends of the filament can be determined as

$$
\tilde{F}=\frac{d \widetilde{H}}{d \varepsilon}=\tilde{\kappa}_{S} \varepsilon-\frac{\pi^{2}}{4} \tilde{\kappa}_{S} \frac{\int_{-\infty}^{\infty} \ldots \int_{-\infty}^{\infty} \sum_{n} n^{2}\left(a_{n}^{2}+b_{n}^{2}\right) e^{-\widetilde{U}} d a_{1} \ldots d a_{n} d b_{1} \ldots d b_{n}}{\int_{-\infty}^{\infty} \ldots \int_{-\infty}^{\infty} e^{-\widetilde{U}} d a_{1} \ldots d a_{n} d b_{1} \ldots d b_{n}}
$$

Notice that a positive value of $\tilde{F}$ means that the force is compressive. Of course, here it is assumed that the filament can deflect in both $y$ and $z$ directions. For a filament confined within a plane, i.e. it can only deflect in one direction, the force can be calculated by simply setting all $b_{i}$ to zero in Eq. (4) and dropping integrations over them in Eq. (5). Now, the task that remains is the evaluation of the integrals that appear in Eq. (5).

\section{Results and Discussions}

Buckling of stiff filament in 2D

Let's proceed by neglecting the entropy contribution first. In this case, equilibrium is achieved when the total elastic energy $\widetilde{U}$ (not free energy), defined in Eq. (4), reaches its minimum. As such, the set of variables $a_{n}$, as functions of $\varepsilon$, must satisfy $\frac{\partial \widetilde{U}}{\partial a_{n}}=0$. Once the values of $a_{n}$ are determined, the compressive force acting on the filament can be found as (refer to the Appendix)

$$
\tilde{F}_{S=0}=\frac{d \widetilde{U}}{d \varepsilon}= \begin{cases}\tilde{\kappa}_{s} \varepsilon, & \text { for } \varepsilon<\varepsilon_{c 0} \\ \tilde{F}_{c 0}=\tilde{\kappa}_{b} \pi^{2}, & \text { for } \varepsilon \geq \varepsilon_{c 0}\end{cases}
$$

where $\varepsilon_{c 0}=\tilde{\kappa}_{b} \pi^{2} / \tilde{\kappa}_{s}$ and the subscript " $S=0$ " indicates that the force is obtained by neglecting any entropy effect. Notice that, in its un-normalized form, the maximum compressive force the 
filament can sustain is $F_{c 0}=\frac{\pi^{2} \kappa_{b}}{L^{2}}$, identical to the well-known Euler buckling load. Furthermore, it can be shown that $a_{n}=0$ (for all $n$ ) when $\varepsilon<\varepsilon_{c 0}$ (see Appendix). Hence, our results essentially reduce to predictions from classical stability analysis, that is, filament deflection will take place only when the axial force reaches the Euler buckling load whereas the column is expected to remain straight when the load level is below this critical value.

Of course, if thermal fluctuations are taken into account then the axial force within filament, as a function of the nominal strain $\varepsilon$, will be different from that given in Eq. (6) and must be calculated from Eq. (5). Nevertheless, the Euler buckling load $\tilde{F}_{c 0}\left(=\tilde{\kappa}_{b} \pi^{2}\right)$ and the corresponding buckling strain $\varepsilon_{c 0}\left(=\tilde{\kappa}_{b} \pi^{2} / \tilde{\kappa}_{s}\right)$ are expected to set the force and strain scales of the problem. It turns out that the normalized force $\tilde{F} / \widetilde{F}_{c 0}$ depends only on the normalized strain $\tilde{\varepsilon}=\varepsilon / \varepsilon_{c 0}$ and the parameter $\alpha=\tilde{\kappa}_{s} \varepsilon_{c 0}^{2}$ (refer to the Appendix). Specifically, we have found that as long as $\tilde{\kappa}_{s} \varepsilon_{c 0}^{2}>\sim 0.5$, very accurate results can be obtained by considering the first deflection mode only. Notice that $\tilde{\kappa}_{S} \varepsilon_{c 0}^{2}=\frac{\pi^{4} \kappa_{b}^{2}}{L^{3} \kappa_{S} k_{B} T}$, so a relatively large value of $\alpha$ corresponds to a short (and hence stiff) filament. For example, $\alpha$ can be estimated to be around 1 for a $160 \mathrm{~nm}$ long Factin, whose persistence length and diameter are taken as $10 \mu \mathrm{m}$ and $8 \mathrm{~nm}$ (Boal, 2002), respectively. The first mode solution can be found as

$$
\frac{\widetilde{\mathrm{F}}}{\widetilde{\mathrm{F}}_{\mathrm{c} 0}}=\tilde{\varepsilon}-\frac{2^{3 / 4} \mathrm{e}^{\alpha(1-\tilde{\varepsilon})^{2} / 4}}{\pi \sqrt{\alpha}\left[\alpha(1-\tilde{\varepsilon})^{2}\right]^{1 / 4}} \frac{\Gamma(3 / 4){ }_{1} \mathrm{~F}_{1}\left(-1 / 4,1 / 2,-\alpha(1-\tilde{\varepsilon})^{2} / 2\right)+\sqrt{2 \alpha(1-\tilde{\varepsilon})^{2}} \Gamma(5 / 4)_{1} \mathrm{~F}_{1}\left(1 / 4,3 / 2,-\alpha(1-\tilde{\varepsilon})^{2} / 2\right)}{\mathrm{I}_{1 / 4}\left(\alpha(1-\tilde{\varepsilon})^{2} / 4\right)+\mathrm{I}_{-1 / 4}\left(\alpha(1-\tilde{\varepsilon})^{2} / 4\right)}
$$

where $\Gamma$ is the Gamma function, ${ }_{1} \mathrm{~F}_{1}$ and $I$ stand for the hypergeometric function and the modified Bessel function of the first kind respectively. Choosing $\tilde{\kappa}_{s} \varepsilon_{c 0}^{2}=1$, the force evolution predicted by Eq. (7) is shown in Fig. 2. Direct calculations by taking two or three modes into account have also been conducted and the results were found to be indistinguishable to the one mode solution. 


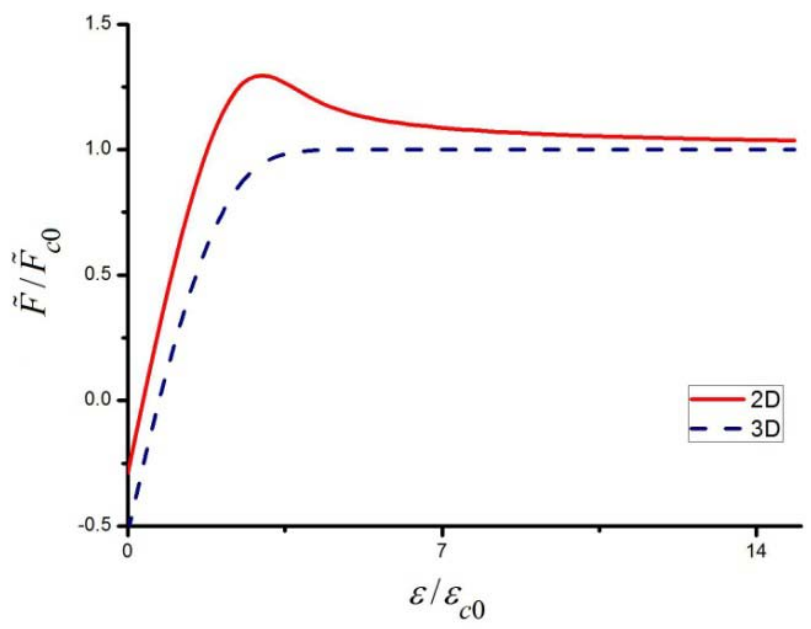

Figure 2. Force as a function of nominal strain in a 2D or 3D filament.

Form Fig. 2, it is clear that the maximum force a 2D filament can sustain is greater than the Euler buckling load. Furthermore, we found that this maximum force, as well as the strain at which this maximum is achieved, take the following forms (refer to the Appendix)

$$
\frac{\tilde{F}_{\max }}{\tilde{F}_{c 0}}=1+\frac{0.41827}{\sqrt{2 \widetilde{\kappa}_{s} \varepsilon_{c 0}^{2}}}, \quad \frac{\varepsilon_{\max }}{\varepsilon_{c 0}}=1+\frac{2.8091}{\sqrt{2 \widetilde{\kappa}_{s} \varepsilon_{c 0}^{2}}} .
$$

Comparison between predictions from Eq. (8) and numerical results by choosing different deflection modes is shown in Fig. 3, which clearly demonstrates that Eq. (8) is very accurate as long as $\tilde{\kappa}_{\mathrm{s}} \varepsilon_{\mathrm{c} 0}^{2}>0.5$. Notice that Eq. (8) also implies that the buckling load of a 2D filament elevates with $\sqrt{T}$. In comparison, Baczynski et al. (2007) examined similar problem, without considering the extensibility of the filament, and concluded that the buckling force increases linearly with temperature. 


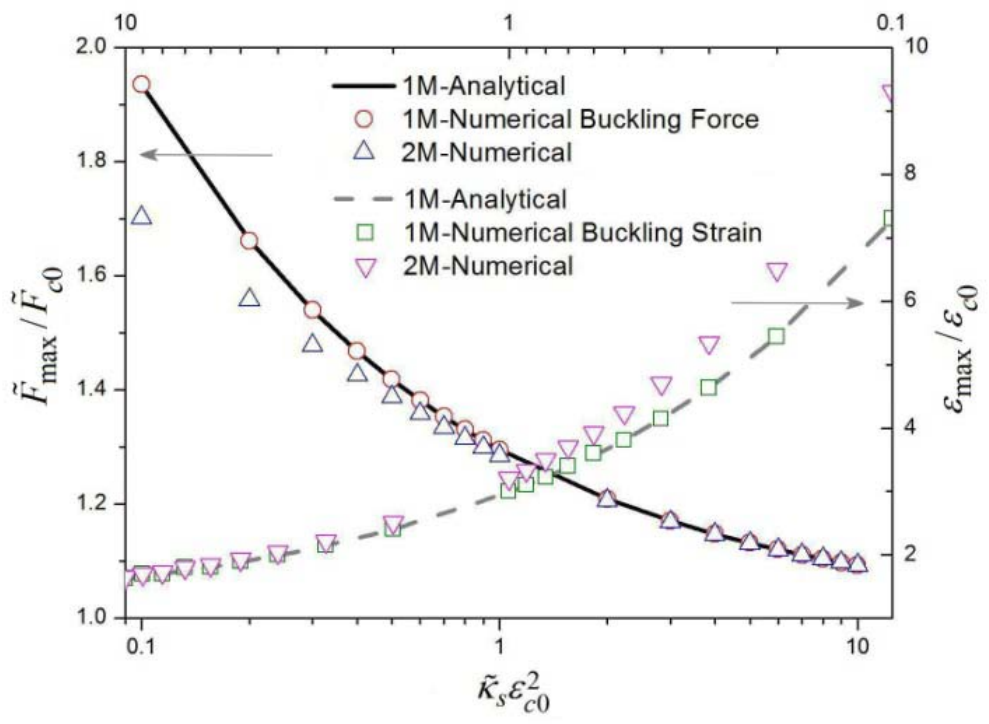

Figure 3. The maximum force, as well as the corresponding strain, as a function of $\tilde{\kappa}_{s} \varepsilon_{c 0}^{2}$. Analytical results are from Eq. (8) while numerical results are obtained by taking into account one or two deflection modes.

\section{Buckling of stiff filament in 3D}

For a filament in $3 \mathrm{D}$, the one mode solution is

$$
\frac{\tilde{F}}{\tilde{F}_{c 0}}=1-\sqrt{\frac{2}{\pi}} \frac{\exp \left[-\alpha(1-\tilde{\varepsilon})^{2} / 2\right]}{\sqrt{\alpha} \operatorname{erfc}[\sqrt{\alpha / 2}(1-\tilde{\varepsilon})]}
$$

$\tilde{F}$ as a function of strain is shown in Fig. 2 for $\tilde{\kappa}_{s} \varepsilon_{c 0}^{2}=1$. An immediate observation from the plot, as well as from Eq. (9), is that the force within the filament will always be less than the Euler buckling load, in direct contrast to the two dimensional case. Physically, this suggests that thermal fluctuations tend to weaken the filament under compression. However, if a filament is confined to deform within a plane, then the constraint imposed on the filament will strengthen it and furthermore this strengthening will out-weigh the weakening induced by thermal excitations which eventually leads to an elevated buckling force in 2D. We must point out that the same conclusion, i.e. the maximum force a 3D filament can sustain is lower than the classical Euler buckling load, has also been obtained by Odijk (1998) via a harmonic analysis as well as recently 
by Blundell and Terentjev (2009) via a mean-field approach. However, to the best of our knowledge, Eq. (9) is the first exact formula for describing how the force within a 3D filament evolves as compression increases. In addition, our theory provides a seamless connection between two and three dimensional cases and clearly demonstrates their differences.

\section{Stretching of soft filament in 3D}

In this section, we shift our attention to the stretching of long and flexible filaments. Since $\tilde{\kappa}_{s} \varepsilon_{c 0}^{2}$ is expected to be much less than 1 in this case, we have to consider the contributions from higher order deflection modes. It turns out that the solution can be expressed as

$$
\frac{\tilde{F}}{\tilde{F}_{c 0}}=1-\frac{3}{\exp [3 \alpha(\tilde{\varepsilon}-5 / 2)] \cdot h(\alpha, \tilde{\varepsilon})-1},
$$

where $h(\alpha, \tilde{\varepsilon})$ is a complicated function whose form is given in the Appendix. One quantity we can immediately calculate from Eq. (10) is the force at full extension, that is $\tilde{F}_{0}=\tilde{F}(\varepsilon=0)$. Since the filament is treated as extensible here, $\widetilde{F}_{0}$ will be finite, in contrast to predictions from the FJC and WLC models. Surprisingly, despite the complexity involved in Eq. (10), we found that this force can be well approximated by

$$
\frac{\widetilde{F}_{0}}{\widetilde{F}_{c 0}}=-\frac{1.259}{\left(\widetilde{\kappa}_{S} \varepsilon_{c 0}^{2}\right)^{2 / 3}},
$$

as shown in Fig. 4. Notice that the negative sign here represents the fact that the force is tensile. One thing we want to point out is that in reality, depending on the nature of the bio-molecule, $\tilde{F}_{0}$ may not be able to reach the level predicted by Eq. (11) due to force-induced filament failure, a scenario that has not been considered here. 


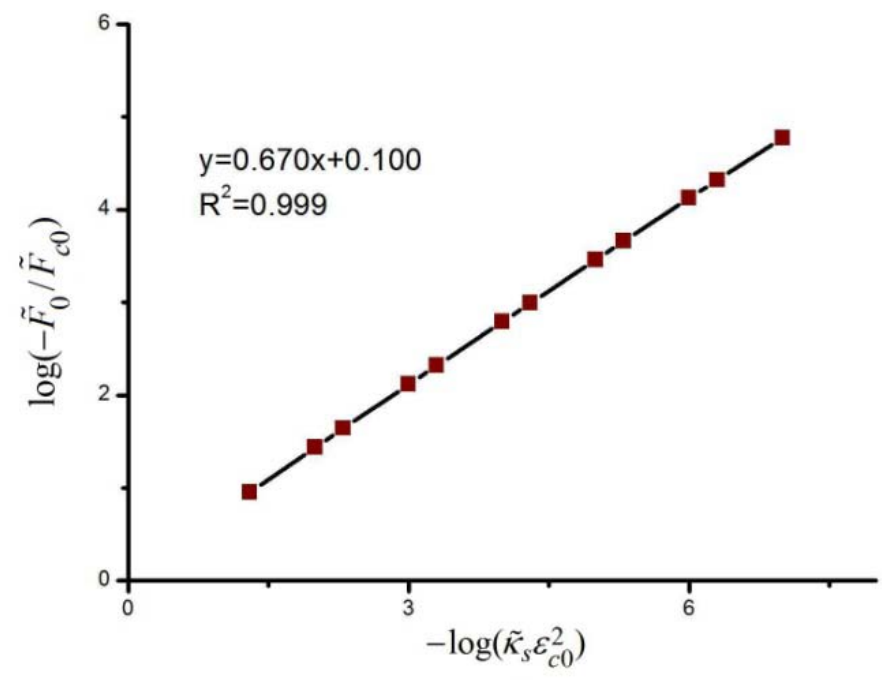

Figure 4. Force at zero strain as a function of $\tilde{\kappa}_{s} \varepsilon_{c 0}^{2}$.

Comparisons between our predictions and several sets of experiments on DNAs or F-actin are shown in Fig. 5. The dsDNA data in Fig. 5(a) is taken from (Wang et al., 1997) with $L \approx 1.3 \mu \mathrm{m}$ and $\kappa_{s} \approx 1246 \mathrm{pN}$ while the two F-actin data, one with $L \approx 11.3 \mu \mathrm{m}, \kappa_{s} \approx 32 \mathrm{nN}$ and the other with $L \approx 19 \mu \mathrm{m}, \kappa_{s} \approx 35 \mathrm{nN}$, are from (Liu and Pollack, 2002). If the persistence lengths of the DNA and the F-actin are taken to be $50 \mathrm{~nm}$ and $8 \mu \mathrm{m}$, respectively, then the values of $\tilde{\kappa}_{s} \varepsilon_{c 0}^{2}$ corresponding to these three sets of data are all of the order of $10^{-7}$. The theoretical values were arrived at by choosing $\tilde{\kappa}_{s} \varepsilon_{c 0}^{2}=4 \times 10^{-7}$. Similarly, the three data sets in Fig. 5(b) are from (Smith et al., 1992), (Smith et al., 1996) and (Baumann et al., 2000), corresponding to long dsDNAs with lengths of $26 \mu \mathrm{m}, 16.4 \mu \mathrm{m}$ and $16.5 \mu \mathrm{m}$, respectively, and, consequently, values of $\tilde{\kappa}_{s} \varepsilon_{c 0}^{2}$ of the order of $10^{-10}$. Hence, the theoretical prediction plotted in Fig. 5(b) was based on $\tilde{\kappa}_{s} \varepsilon_{c 0}^{2}=2 \times 10^{-10}$. Clearly, our theory is capable of explaining all the data well.

For practical purposes, it might be desirable that a simple force-extension relationship, instead of the complicated one shown in Eq. (10), can be derived. In light of Eq. (11), we found that Eq. (10) can be well approximated by (see the Appendix for details) 


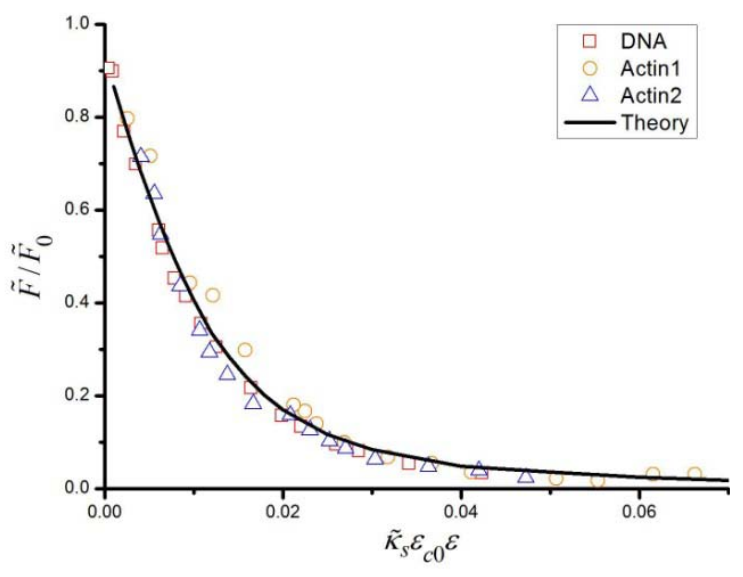

(a)

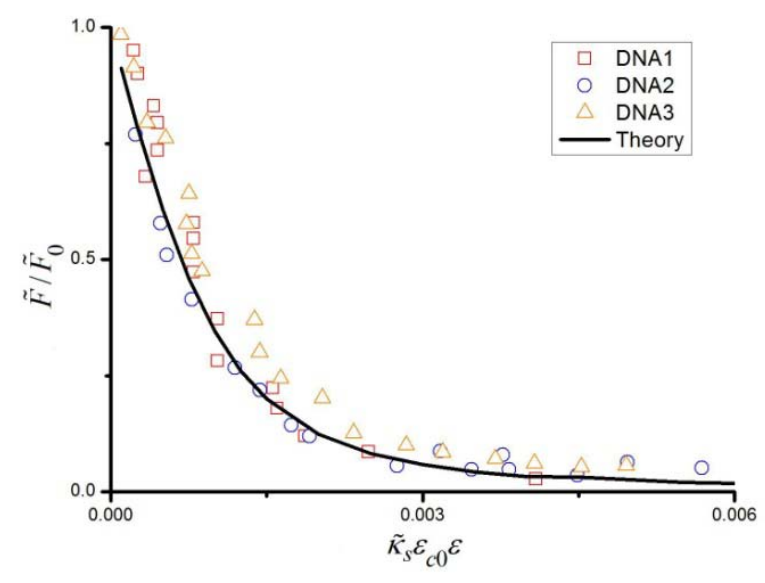

(b)

Figure 5. Comparison between theory and experimental data. (a)- Theoretical prediction, with $\tilde{\kappa}_{s} \varepsilon_{c 0}^{2}=4 \times 10^{-7}$, versus data on short DNA (Wang et al., 1997) and long F-actin (Liu and Pollack, 2002); (b)- Theoretical prediction, with $\tilde{\kappa}_{s} \varepsilon_{c 0}^{2}=2 \times 10^{-10}$, versus data on long DNAs (Smith et al., 1992; Smith et al., 1996; Baumann et al., 2000). Notice that, since $\widetilde{F}_{0}$ is negative, refer to Eq. (11), a positive value of $\tilde{F} / \widetilde{F}_{0}$ here means that $\tilde{F}$ is also negative, i.e. this force is tensile.

$$
\frac{\varepsilon}{\varepsilon_{c 0}}=\frac{1.412}{\widetilde{\kappa}_{s} \varepsilon_{c 0}^{2}}\left(\frac{1}{-\tilde{F} / \tilde{F}_{c 0}}\right)^{1 / 2}+\frac{\tilde{F}}{\tilde{F}_{c 0}},
$$

which has the same scaling form as that proposed in (Odijk, 1995), however the constant, i.e. 1.412, here is different from that derived by Odijk (1995). Eq. (12) suggests that, when $\widetilde{F}$ is relatively small (compared to $\widetilde{F}_{0}$ ), we should have the following scaling relation

$$
\frac{\tilde{F}}{\tilde{F}_{c 0}}=-\left(\frac{1.412}{\widetilde{\kappa}_{s} \varepsilon_{c 0}^{2}}\right)^{2}\left(\frac{\varepsilon_{c 0}}{\varepsilon}\right)^{2}, \text { or } \frac{F \kappa_{b}}{\left(k_{B} T\right)^{2}}=-0.2 \frac{1}{\varepsilon^{2}}
$$

To test the validity of Eq. (13), its prediction was compared to the exact solution and the result is shown in Fig. 6. Obviously, as expected, Eq. (13) is indeed very accurate when the force is small (note that $\log \left[1.412^{2}\right]=0.3$ ). Eq. (13) also shows that the force will elevate with $1 / \varepsilon^{2}$ as the filament is stretched close to its contour length, a well-known result previously obtained by 
Marko and Siggia (1995). However, we must point out that the tensile force predicted here is $\sim 20 \%$, rather significantly lower than that from the WLC model (Marko and Siggia, 1995).

Several factors may contribute to such discrepancy, for one thing the axial stretching of filament has been considered in our analysis which leads to the appearance of quartic terms of the amplitudes of each deflection mode in the energy expression as shown in Eq. (4). In contrast, the elastic energy in the WLC formulation contains only quadratic terms of these random variables because the bio-polymer is assumed to be inextensible. In addition, undulations of the filament in two transverse directions are coupled in our model, refer to Eq. (4), whereas they were essentially treated as independent in (Marko and Siggia, 1995).

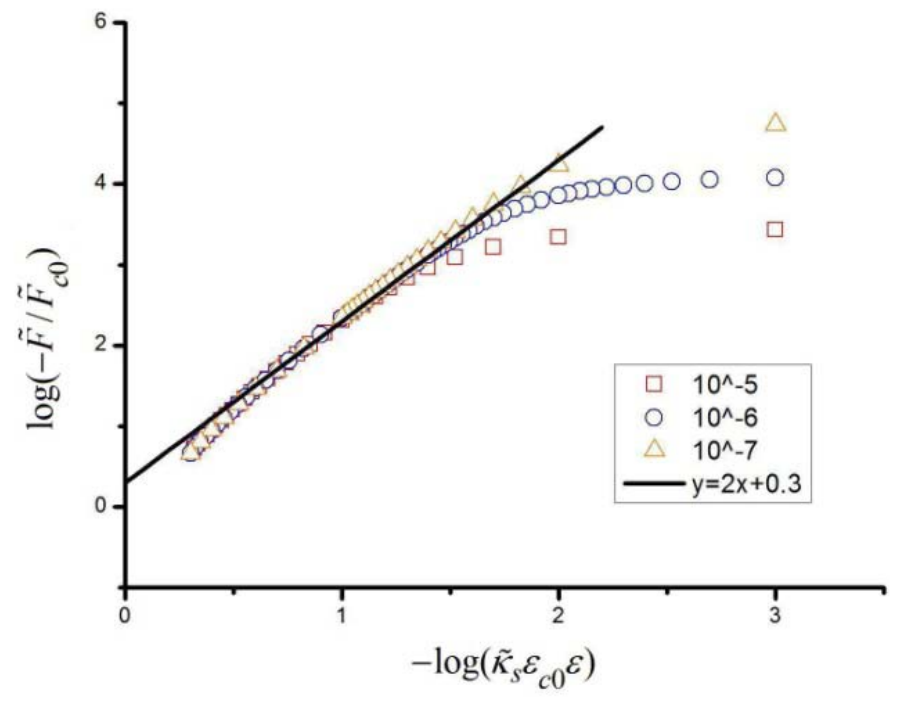

Figure 6. Evolution of filament force with respect to the nominal strain. The solid line represents the prediction by Eq. (13) while the symbols correspond to exact solutions obtained from Eq. (10).

\section{Conclusions}

In this paper, we present a unified theory for examining the behaviour of filaments under stretching as well as compression, an issue of fundamental significance in, for example, cytoskeleton mechanics and polymer physics. By considering the stretching and bending of the 
filament simultaneously, we are able to obtain closed form force-extension relationships predicting how stiff filaments buckle as well as how a flexible filament behaves when stretched close to its contour length. The main findings of this study are summarized as follows.

- The maximum force a 2D filament can sustain is found to be higher than the Euler buckling load which suggests that the filament is strengthened by the constraint imposed. In addition, analytical expression of this buckling force, as well as the strain at which this maximum load is achieved, has also been derived.

- In comparison, we found that the force in a 3D filament is always lower than the Euler buckling load suggesting that thermal fluctuations weaken the filament under compression.

- Closed form relationship has been obtained to predict how force increases when a flexible filament is close to being fully stretched. The theory proposed here is capable of explaining a variety of experimental observations rather well.

- From the point of view of scaling, our theory is consistent with several well-known results such as that obtained by Odijk (1995) or by Marko and Siggia (1995). However, in comparison with our results, those models seem to over-predict the filament force considerably.

We believe that findings obtained here might be important in future investigations on the mechanical response of branched actin networks, commonly formed in the leading edge of motile cells, which consist of short filaments with lengths in the range of $100 \mathrm{~nm}-1 \mu \mathrm{m}$ (Fletcher and Mullins, 2010). Specifically, it has been reported that, when under compression, such highly organized network will actually undergo stress softening (Chaudhuri et al., 2007). Furthermore, it was found that such a softening stage is completely reversible suggesting that buckling of individual F-actin filaments may play an important role there. As such, our results on the buckling of stiff bio-polymers in $3 \mathrm{~d}$ could be very useful in explaining or predicting how these branched networks behave. For instance, it might be possible to utilize Eq. (9) or similar 
relationships obtained under different boundary conditions as input functions, describing the behavior of individual filaments, in simulating the bulk response of such actin networks which could greatly reduce the computation cost. In addition, recent advances in technology have enabled researchers to grow and then manipulate individual bio-filaments confined between two coverslips. For example, forces developed by a polymerizing F-actin (Berro et al., 2007) and a growing microtubule (Janson and Dogterom, 2004; Kerssemakers et al., 2003) have all been estimated by analyzing the two-dimensional buckling of these filaments. It is conceivable that Eq. (11) and (12) obtained here, describing the compressive response of stiff bio-polymers in 2d, could be used to guide the design as well as interpret the data of similar experiments in the future. Finally, the force-extension relationship of long and flexible filaments derived in this study could serve as a building block for future constitutive modelling (Arruda and Boyce, 1993; Gardel et al., 2004; Storm et al., 2005; Palmer and Boyce, 2008), as well as numerical simulation (Onck et al., 2005; van Dillen et al., 2008; Chen and Shenoy, 2010), of different polymer materials where the phenomenon of stress stiffening is thought to be directly linked to the elevated force needed to fully stretch a polymer molecule.

\section{Acknowledgement}

Y.L. is grateful for support from the Research Grants Council (Project No. HKU 7148/10E ) of the Hong Kong Special Administration Region as well as a University Development Fund from the University of Hong Kong. V.B.S. acknowledges support through a grant from the National Science Foundation (No. CMMI-1129172). 


\section{References}

1. Arruda, E.M. and M.C. Boyce. 1993. A three-dimensional constitutive model for the large stretch behavior of rubber elastic materials. J. Mech. Phys. Solids 41: 389-412.

2. Baczynski, K., R. Lipowsky and J. Kierfeld. 2007. Stretching of buckled filaments by thermal fluctuations. Physical Review E 76: 61914.

3. Baumann, C.G., V.A. Bloomfield, S.B. Smith, C. Bustamante, M.D. Wang and S.M. Block. 2000. Stretching of single collapsed DNA molecules. Biophysical Journal 78: 1965-1978.

4. Berro, J., A. Michelot, L. Blanchoin, D.R. Kovar and J.-L. Martiel. 2007. Attachment conditions control actin filament buckling and the production of forces. Biophysical Journal 92: 2546-2558.

5. Blundell, J. and E. Terentjev. 2009. Buckling of semiflexible filaments under compression. Soft Matter 5: 4015-4020.

6. Boal, D. 2002. Mechanics of the Cell. Cambridge University Press, Cambridge.

7. Chaudhuri, O., S.H. Parekh and D.A. Fletcher. 2007. Reversible stress softening of actin networks. Nature 445: 295-298.

8. Chen, P. and V.B. Shenoy. Strain stiffening induced by molecular motors in active crosslinked biopolymer networks. Soft Matter 6: 3548-3561.

9. Dill, K.A. and S. Bromberg. 2002. Molecular Driving Forces: Statistical Thermodynamics in Chemistry and Biology. Garland Science, New York.

10. Eggert, U.S., T.J. Mitchison and C.M. Field. 2006. Animal cytokinesis: From parts list to mechanisms. Annu. Rev. Biochem. 75: 543-566. 
11. Fletcher, D.A. and R.D. Mullins. 2010. Cell mechanics and the cytoskeleton. Nature 463: 485-492.

12. Gardel, M.L., J.H. Shin, F.C. MacKintosh, L. Mahadevan, P. Matsudaira and D.A. Weitz. 2004. Elastic behaviour of cross-linked and bundled actin network. Science 304: 1301-1305.

13. Gholami, A., J. Wilhelm and E. Frey. 2006. Entropic forces generated by grafted semiflexible polymers. Physical Review E 74: 41803.

14. Gittes, F., B. Mickey, J. Nettleton and J. Howard. 1993. Flexural rigidity of microtubules and actin filaments measured from thermal fluctuations in shape. Journal of Cell Biology 120: 923934.

15. Isambert, H., P. Venier, A.C. Maggs, A. Fattoum, R. Kassab, D. Pantaloni and M.F. Carlier. 1995. Flexibility of actin filaments derived from thermal fluctuations. Effect of bound nucleotide, phalloidin, and muscle regulatory proteins. The Journal of Biological Chemistry 270: 1143711444.

16. Janson, M.E. and M. Dogterom. 2004. Scaling of microtubule force-velocity curves obtained at different tubulin concentrations. Physical Review Letters 92: 248101.

17. Kerssemakers, J.W.J., M.E. Janson, A. van der Horst and M. Dogterom. 2003. Optical trap setup for measuring microtubule pushing force. Applied Physics Letters 83: 4441.

18. Lee, N.-K., A. Johner and S.-C. Hong. 2007. Compressing a rigid filament: Buckling and cyclization. Eur. Phys. J. E. 24: 229-241.

19. Li, T. 2008. A mechanics model of microtubule buckling in living cells, Journal of Biomechanics 41: 1722-1729. 
20. Liu, X. and G.H. Pollack. 2002. Mechanics of F-actin characterized with microfabricated cantilevers. Biophysical Journal 83: 2705-2715.

21. MacKintosh, F.C. 2006. Polymer-based models of cytoskeletal networks. Cytoskeletal mechanics: models and measurements, M. Mofrad and R. Kamm, Editors. Cambridge University Press: Cambridge. 152-169.

22. Marko, J.F. and E.D. Siggia. 1995. Stretching DNA. Marcomolecules 28: 8759-8770.

23. Odijk, T. 1995. Stiff chains and filaments under tension. Macromolecules 28: 7016-7018.

24. Odijk, T. 1998. Microfibrillar buckling within fibers under compression. Journal of Chemical Physics 108: 6923-6928.

25. Onck, P.R., T. Koeman, T. van Dillen and E. van der Giessen. 2005. Alternative explanation of stiffening in cross-linked semiflexible networks. Physical Review Letters 95: 178102.

26. Palmer, J.S. and M.C. Boyce. 2008. Constitutive modelling of the stress-strain behaviour of F-actin filament network. Acta Biomaterialia 4: 597-612.

27. Pollard, T.D. and G.G. Borisy. 2003. Cellular motility driven by assembly and disassembly of actin filaments. Cell 112: 453-465.

28. Smith, S.B., Y. Cui and C. Bustamante. 1996. Overstretching B-DNA: the elastic response of individual double-stranded and single-stranded DNA molecules. Science 271: 795-799.

29. Smith, S.B., L. Finzi and C. Bustamante. 1992. Direct mechanical measurements of the elasticity of single DNA molecules by using magnetic beads. Science 258: 1122-1126.

30. Storm, C., J.J. Pastore, F.C. MacKintosh, T.C. Lubensky and P.A. Janmey. 2005. Nonlinear elasticity in biological gels. Nature 435: 191-194. 
31. Su, T. and P.K. Purohit. 2009. Mechanics of forced unfolding of proteins. Acta Biomaterialia 5: $1855-1863$.

32. van Dillen, T., P.R. Onck and E. van der Giessen. 2008. Models for stiffnening in crosslinked biopolymer networks: A comparative study. J. Mech. Phys. Solids 56: 2240-2264.

33. Wang, M.D., H. Yin, R. Landick, J. Gelles and S.M. Block. 1997. Stretching DNA with optical tweezers. Biophysical Journal 72: 1335-1346. 


\section{Appendix}

\section{Compressive response of a filament when entropy effect is neglected}

Since the contribution of entropy is neglected here, equilibrium is achieved when the total elastic energy $\widetilde{U}$ defined in (4), reaches its minimum. As such, the set of variables $a_{n}$, as functions of $\varepsilon$, must satisfy

$$
\frac{\partial \widetilde{U}}{\partial a_{n}}=a_{n}\left[\frac{1}{2} \tilde{\kappa}_{b} n^{4} \pi^{4}+\frac{\pi^{2}}{2} n^{2} \tilde{\kappa}_{s}\left(-\varepsilon+\frac{\pi^{2}}{4} \sum_{m} m^{2} a_{m}^{2}\right)\right]=0
$$

Hence, the only admissible combinations of $a_{n}$ are

$$
a_{n}=0, n=1,2,3 \ldots
$$

or

$$
\left\{\begin{array}{cl}
\frac{1}{2} \tilde{\kappa}_{b} n^{4} \pi^{4}+\frac{\pi^{2}}{2} n^{2} \tilde{\kappa}_{s}\left(-\varepsilon+\frac{\pi^{2}}{4} n^{2} a_{n}^{2}\right)=0, & \text { for arbitrary } n \\
a_{m}=0, & \text { for } m \neq n
\end{array},\right.
$$

from which a critical nominal strain level can be identified as $\varepsilon_{c 0}=\tilde{\kappa}_{b} \pi^{2} / \tilde{\kappa}_{s}$. For $\varepsilon<\varepsilon_{c 0}$, the minimum energy configuration is achieved by

$$
a_{n}=0, n=1,2,3 \ldots ; \quad \widetilde{U}=\frac{1}{2} \tilde{\kappa}_{s} \varepsilon^{2},
$$

suggesting that the filament will remain straight if the nominal strain is below $\varepsilon_{c 0}$. However, for $\varepsilon \geq \varepsilon_{c 0}$, minimization of $\widetilde{U}$ leads to

$$
\frac{\pi^{2}}{4} a_{1}^{2}=\varepsilon-\varepsilon_{c 0}, a_{m}=0 \text { for } m>1 ; \quad \widetilde{U}=\tilde{\kappa}_{b} \pi^{2}\left(\varepsilon-\varepsilon_{c 0}\right)+\frac{1}{2} \tilde{\kappa}_{s} \varepsilon_{c 0}^{2}
$$

i.e. deflection (or buckling) of filament will take place under such circumstance. At this point, it is clear that the compressive force acting on the filament takes the following form

$$
\tilde{F}_{S=0}=\frac{d \widetilde{U}}{d \varepsilon}= \begin{cases}\tilde{\kappa}_{S} \varepsilon, & \text { for } \varepsilon<\varepsilon_{c 0} \\ \tilde{F}_{c 0}=\tilde{\kappa}_{b} \pi^{2}, & \text { for } \varepsilon \geq \varepsilon_{c 0}\end{cases}
$$


where the subscript " $S=0$ " indicates that the force is obtained by neglecting any entropy effect. Notice that the maximum compressive force the filament can sustain, in its un-normalized form, is $F_{c 0}=\frac{\pi^{2} \kappa_{b}}{L^{2}}$ which is exactly the classical Euler buckling load.

Buckling force of a 2D filament immersed in a thermal bath

If only the first mode is considered, then according to (5) the filament force can be calculated as

$$
\frac{\tilde{F}}{\tilde{F}_{c 0}}=\frac{\varepsilon}{\varepsilon_{c 0}}-\frac{1}{\varepsilon_{c 0}} \frac{\int_{-\infty}^{\infty} a^{2} e^{-\widetilde{U}} d a}{\int_{-\infty}^{\infty} e^{-\widetilde{U}} d a} \quad, \quad \widetilde{U}=\tilde{\kappa}_{s}\left(\frac{1}{2} a^{4}+\left(\varepsilon_{c 0}-\varepsilon\right) a^{2}\right)
$$

The maximum force is achieved when $\frac{\partial \tilde{F}}{\partial \varepsilon}=0$, which leads to

$$
1-\left.\frac{\left(\int_{-\infty}^{\infty} a^{2} e^{-\widetilde{U}} d a\right)^{\prime}\left(\int_{-\infty}^{\infty} e^{-\widetilde{U}} d a\right)-\left(\int_{-\infty}^{\infty} a^{2} e^{-\widetilde{U}} d a\right)\left(\int_{-\infty}^{\infty} e^{-\widetilde{U}} d a\right)}{\left(\int_{-\infty}^{\infty} e^{-\widetilde{U}} d a\right)^{2}}\right|_{\varepsilon=\varepsilon_{\max }}=0 .
$$

Notice that the Taylor series expansion of $e^{-\widetilde{U}}$ at $\varepsilon=\varepsilon_{c 0}$ is

$$
e^{-\widetilde{U}}=\sum_{n=0}^{\infty} \frac{\left[\widetilde{\kappa}_{s}\left(\varepsilon-\varepsilon_{c 0}\right)\right]^{n}}{n !} a^{2 n} e^{-\frac{\widetilde{\kappa}_{s}}{2} a^{4}}
$$

from which Eq. (A7) can be rewritten as

$$
\frac{1}{2}=\frac{\sum_{n=0}^{\infty} \frac{\Gamma\left(\frac{2 n+5}{4}\right)}{n !}\left[\sqrt{2 \widetilde{\kappa}_{S}}\left(\varepsilon-\varepsilon_{c 0}\right)\right]^{n}}{\sum_{n=0}^{\infty} \frac{\Gamma\left(\frac{2 n+1}{4}\right)}{n !}\left[\sqrt{2 \widetilde{\kappa}_{S}}\left(\varepsilon-\varepsilon_{c 0}\right)\right]^{n}}-\left.\left(\frac{\sum_{n=0}^{\infty} \frac{\Gamma\left(\frac{2 n+3}{4}\right)}{n !}\left[\sqrt{2 \widetilde{\kappa}_{S}}\left(\varepsilon-\varepsilon_{c 0}\right)\right]^{n}}{\sum_{n=0}^{\infty} \frac{\Gamma\left(\frac{2 n+1}{4}\right)}{n !}\left[\sqrt{2 \widetilde{\kappa}_{S}}\left(\varepsilon-\varepsilon_{c 0}\right)\right]^{n}}\right)^{2}\right|_{\varepsilon=\varepsilon_{\max }} .
$$

This equation can be generalized as

$$
\frac{\sum_{n=0}^{\infty} \frac{\Gamma\left(\frac{2 n+5}{4}\right)}{n !} x^{n}}{\sum_{n=0}^{\infty} \frac{\Gamma\left(\frac{2 n+1}{4}\right)}{n !} x^{n}}-\left(\frac{\sum_{n=0}^{\infty} \frac{\Gamma\left(\frac{2 n+3}{4}\right)}{n !} x^{n}}{\sum_{n=0}^{\infty} \frac{\Gamma\left(\frac{2 n+1}{4}\right)}{n !} x^{n}}\right)^{2}=\frac{1}{2}
$$

which has multiple solutions with the smallest one being $x=2.80911$. Therefore (A9) can be solved by

$$
\frac{\varepsilon_{\max }}{\varepsilon_{c 0}}=1+\frac{2.80911}{\sqrt{2 \widetilde{\kappa}_{s} \varepsilon_{c 0}^{2}}}
$$


By substituting (A11) into (A6), the buckling load can be obtained as

$$
\frac{\tilde{F}_{\max }}{\widetilde{F}_{c 0}}=\frac{\varepsilon_{\max }}{\varepsilon_{c 0}}-\frac{1}{\varepsilon_{c 0}} \sqrt{\frac{2}{\widetilde{\kappa}_{s}}} \frac{\sum_{n=0}^{\infty} \frac{\Gamma\left(\frac{2 n+3}{4}\right)}{n !} 2.80911^{n}}{\sum_{n=0}^{\infty} \frac{\Gamma\left(\frac{2 n+1}{4}\right)}{n !} 2.80911^{n}},
$$

which can be further reduced to

$$
\frac{\tilde{F}_{\max }}{\tilde{F}_{c 0}}=1+\frac{0.41827}{\sqrt{2 \widetilde{\kappa}_{s} \varepsilon_{c 0}^{2}}}
$$

\section{Filament force in 3D}

Let $r_{n}^{2}=\frac{\pi^{2}}{4} n^{2}\left(a_{n}^{2}+b_{n}^{2}\right)$, we can convert the integrals in (5) into polar coordinates. Next, replacing $r_{n}^{2}$ with $x_{n}$ leads to

$$
\frac{\tilde{F}}{\tilde{F}_{c 0}}=\frac{\varepsilon}{\varepsilon_{c 0}}-\frac{1}{\varepsilon_{c 0}} \frac{\int_{0}^{\infty} \ldots \int_{0}^{\infty} \sum n x_{n} e^{-\widetilde{U}} d x_{1} \ldots d x_{n}}{\int_{0}^{\infty} \ldots \int_{0}^{\infty} e^{-\widetilde{U}} d x_{1} \ldots d x_{n}}, \quad \widetilde{U}=\tilde{\kappa}_{s}\left[\frac{1}{2}\left(\sum_{n} x_{n}\right)^{2}+\varepsilon_{c 0} \sum_{n} n^{2} x_{n}-\varepsilon \sum_{n} x_{n}\right]
$$

We proceed by making another transformation of variables as $y_{1}=\sum_{1}^{n} x_{n}, y_{2}=x_{2}, \ldots, y_{n}=x_{n}$. As a result, integration over $y_{1}$ can be carried out exactly and the force becomes

$$
\frac{\tilde{F}}{\tilde{F}_{c 0}}=1-\sqrt{\frac{2}{\pi}} \frac{e^{\frac{-\widetilde{\kappa}_{S}\left(\varepsilon-\varepsilon_{c 0}\right)^{2}}{2}}}{\sqrt{\widetilde{\kappa}_{s} \varepsilon_{c 0}^{2}}} \frac{\int_{0}^{\infty} \ldots \int_{0}^{\infty} e^{-\widetilde{\kappa}_{s}\left[\frac{1}{2}\left(\sum_{2}^{n} y_{n}\right)^{2}-\left(\varepsilon-\varepsilon_{c 0}\right) \sum_{2}^{n} y_{n}+\varepsilon_{c 0} \sum_{2}^{n}\left(n^{2}-1\right) y_{n}\right]} d y_{2} \ldots d y_{n}}{\int_{0}^{\infty} \ldots \int_{0}^{\infty} \operatorname{erfc}\left[\sqrt{\frac{\widetilde{\kappa}_{s}}{2}}\left(\sum_{2}^{n} y_{n}-\left(\varepsilon-\varepsilon_{c 0}\right)\right)\right] e^{-\widetilde{\kappa}_{S} \varepsilon_{c 0} \sum_{2}^{n}\left(n^{2}-1\right) y_{n}} d y_{2} \ldots d y_{n}},
$$

from which the one mode solution can be found as

$$
\frac{\tilde{F}}{\tilde{F}_{c 0}}=1-\sqrt{\frac{2}{\pi}} \frac{e^{\frac{-\widetilde{\kappa}_{S}\left(\varepsilon-\varepsilon_{c 0}\right)^{2}}{2}}}{\sqrt{\widetilde{\kappa}_{s} \varepsilon_{c 0}^{2}}} \frac{1}{\operatorname{erfc}\left[-\sqrt{\frac{\widetilde{\kappa}_{s}}{2}\left(\varepsilon-\varepsilon_{c 0}\right)}\right]} .
$$

Similarly, let $z_{2}=\sum_{2}^{n} y_{n}, z_{3}=y_{3}, \ldots, z_{n}=y_{n}$, (A15) can be further reduced to 


$$
\frac{\tilde{F}}{\tilde{F}_{c 0}}=1-
$$

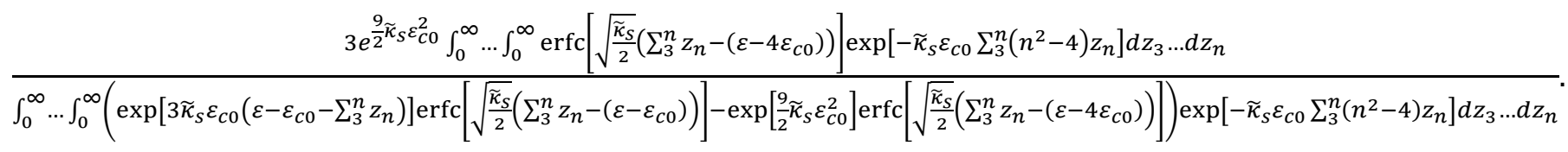

Note that (A17) can be rewritten as

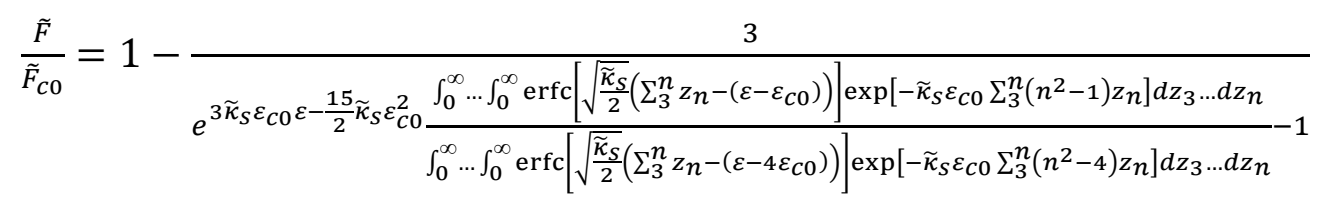

which, surprisingly, can be evaluated exactly as

$$
\frac{\tilde{F}}{\tilde{F}_{c 0}}=1-\frac{3}{e^{3 \widetilde{\kappa}_{S} \varepsilon_{c 0}^{2}\left(\frac{\varepsilon}{\varepsilon_{c 0}}-\frac{5}{2}\right)} \frac{\frac{1}{\prod_{j=1}^{n}\left(j^{2}+4 j+3\right)} \operatorname{erfc}\left[\sqrt{\frac{\widetilde{\kappa}_{S} \varepsilon_{c 0}^{2}}{2}}\left(1-\frac{\varepsilon}{\varepsilon_{c 0}}\right)\right]+a}{\frac{1}{\prod_{j=1}^{n}\left(j^{2}+4 j\right)} \operatorname{erfc}\left[\sqrt{\frac{\widetilde{\kappa}_{S} \varepsilon_{c 0}^{2}}{2}}\left(4-\frac{\varepsilon}{\varepsilon_{c 0}}\right)\right]+b}-1}
$$

With

$$
a=\sum_{i=1}^{n}(-1)^{i} \frac{f(i)}{\prod_{j=i+1}^{n}(j-i)(j+i+4)} \operatorname{erfc}\left[\sqrt{\frac{\tilde{\kappa}_{s} \varepsilon_{c 0}^{2}}{2}}\left((i+2)^{2}-\frac{\varepsilon}{\varepsilon_{c 0}}\right)\right] \exp \left[(i+1)(i+3) \tilde{\kappa}_{s} \varepsilon_{c 0}^{2}\left(\frac{1}{2}+\frac{(i+2)^{2}}{2}-\frac{\varepsilon}{\varepsilon_{c 0}}\right)\right]
$$

and

$$
b=\sum_{i=1}^{n}(-1)^{i} \frac{g(i)}{\prod_{j=i+1}^{n}(j-i)(j+i+4)} \operatorname{erfc}\left[\sqrt{\frac{\widetilde{\kappa}_{\varepsilon_{c 0}^{2}}^{2}}{2}}\left((i+2)^{2}-\frac{\varepsilon}{\varepsilon_{c 0}}\right)\right] \exp \left[i(i+4) \tilde{\kappa}_{s} \varepsilon_{c 0}^{2}\left(2+\frac{(i+2)^{2}}{2}-\frac{\varepsilon}{\varepsilon_{c 0}}\right)\right] .
$$

$f(i)$ and $g(i)$ are functions of $i$ and relate to each other as $g(i)=\frac{(i+2)^{2}-1}{(i+2)^{2}-4} f(i)$. To determine these functions, we first realize that (A14) can be rewritten as 


$$
\begin{aligned}
& \frac{\tilde{F}}{\tilde{F}_{c 0}}=
\end{aligned}
$$

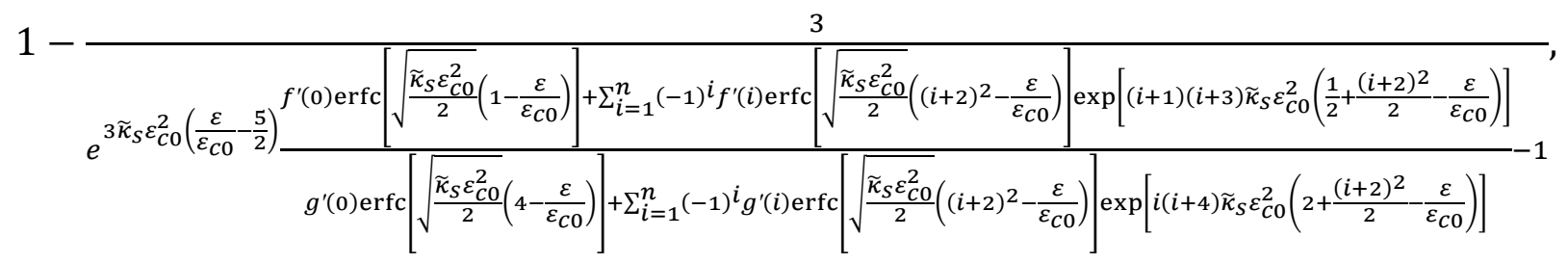

where, for $i \geq 1, f^{\prime}(i)$ and $g^{\prime}(i)$ relate to $f(i)$ and $g(i)$ as

$$
f^{\prime}(i)=f(i) \frac{\prod_{j=1}^{n}\left(j^{2}+4 j\right)}{\prod_{j=i+1}^{n}(j-i)(j+i+4)}, \quad g^{\prime}(i)=g(i) \frac{\prod_{j=1}^{n}\left(j^{2}+4 j\right)}{\prod_{j=i+1}^{n}(j-i)(j+i+4)} .
$$

In addition, as $n \rightarrow \infty$, we found

$$
g^{\prime}(i)-f^{\prime}(i)=\frac{(i+2)^{2}}{4}
$$

Therefore, the asymptotic expressions of $f^{\prime}(i)$ and $g^{\prime}(i)$, as $n \rightarrow \infty$, can be determined as

$$
\begin{aligned}
& f^{\prime}(0)=\frac{1}{4}, \quad f^{\prime}(i)=\frac{(i+2)^{4}-4(i+2)^{2}}{12} \text { for } i \geq 1, \\
& g^{\prime}(0)=1, \quad g^{\prime}(i)=\frac{(i+2)^{4}-(i+2)^{2}}{12} \text { for } i \geq 1
\end{aligned}
$$

from which the expressions of $f(i)$ and $g(i)$ can be obtained as

$$
\begin{aligned}
& f(i)=\frac{(i+2)^{4}-4(i+2)^{2}}{12} \lim _{n \rightarrow \infty} \frac{\prod_{j=i+1}^{n}(j-i)(j+i+4)}{\prod_{j=1}^{n}\left(j^{2}+4 j\right)}=\frac{(i+2)^{4}-4(i+2)^{2}}{12} \prod_{j=1}^{i} \frac{1}{(2 j+3)(2 j+4)}, \\
& g(i)=\frac{(i+2)^{4}-(i+2)^{2}}{12} \lim _{n \rightarrow \infty} \frac{\prod_{j=i+1}^{n}(j-i)(j+i+4)}{\prod_{j=1}^{n}\left(j^{2}+4 j\right)}=\frac{(i+2)^{4}-(i+2)^{2}}{12} \prod_{j=1}^{i} \frac{1}{(2 j+3)(2 j+4)} .
\end{aligned}
$$

Notice that $f(i)$ and $g(i)$ are independent of $n$. Now we are able to obtain the general expressions of $f^{\prime}(i)$ and $g^{\prime}(i)$ as 


$$
\begin{array}{ll}
f^{\prime}(0)=\frac{\prod_{j=1}^{n}\left(j^{2}+4 j\right)}{\prod_{j=1}^{n}\left(j^{2}+4 j+3\right)}, & f^{\prime}(i)=\frac{(i+2)^{4}-4(i+2)^{2}}{12} \frac{\prod_{j=1}^{n}\left(j^{2}+4 j\right)}{\prod_{j=1}^{i}(2 j+3)(2 j+4) \prod_{j=i+1}^{n}(j-i)(j+i+4)} \text { for } i \geq 1, \\
g^{\prime}(0)=1, & g^{\prime}(i)=\frac{(i+2)^{4}-(i+2)^{2}}{12} \frac{\prod_{j=1}^{n}\left(j^{2}+4 j\right)}{\prod_{j=1}^{i}(2 j+3)(2 j+4) \prod_{j=i+1}^{n}(j-i)(j+i+4)} \text { for } i \geq 1 .
\end{array}
$$

Substituting (A21) into (A20), finally, the force can be expressed as

$$
\frac{\tilde{F}}{\tilde{F}_{c 0}}=1-\frac{3}{\exp \left[3 \widetilde{\kappa}_{s} \varepsilon_{c 0}^{2}\left(\varepsilon / \varepsilon_{c 0}-5 / 2\right)\right] \cdot h\left(\widetilde{\kappa}_{s} \varepsilon_{c 0}^{2}, \varepsilon / \varepsilon_{c 0}\right)-1},
$$

where

$h\left(\tilde{\kappa}_{s} \varepsilon_{c 0}^{2}, \varepsilon / \varepsilon_{c 0}\right)=$

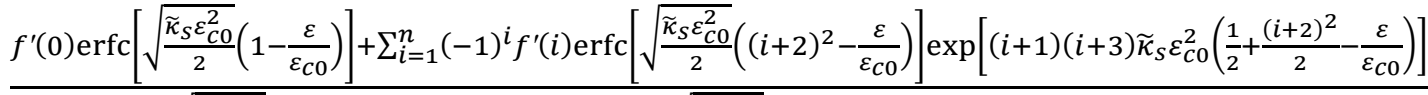

$g^{\prime}(0) \operatorname{erfc}\left[\sqrt{\frac{\widetilde{\kappa}_{s} \varepsilon_{c 0}^{2}}{2}}\left(4-\frac{\varepsilon}{\varepsilon_{c 0}}\right)\right]+\sum_{i=1}^{n}(-1)^{i} g^{\prime}(i) \operatorname{erfc}\left[\sqrt{\frac{\widetilde{\kappa}_{s} \varepsilon_{c 0}^{2}}{2}}\left((i+2)^{2}-\frac{\varepsilon}{\varepsilon_{c 0}}\right)\right] \exp \left[i(i+4) \widetilde{\kappa}_{s} \varepsilon_{c 0}^{2}\left(2+\frac{(i+2)^{2}}{2}-\frac{\varepsilon}{\varepsilon_{c 0}}\right)\right]$

with $f^{\prime}(i)$ and $g^{\prime}(i)$ given by (A21). Of course (A22) is rather complicated and might not be so convenient to use, so an approximate formula, i.e. (12), was proposed here. Comparison between predictions from (12) and the exact solutions, given by (A22), is shown in Fig. A1, which clearly demonstrates that (12) is indeed an excellent approximation of (A22).

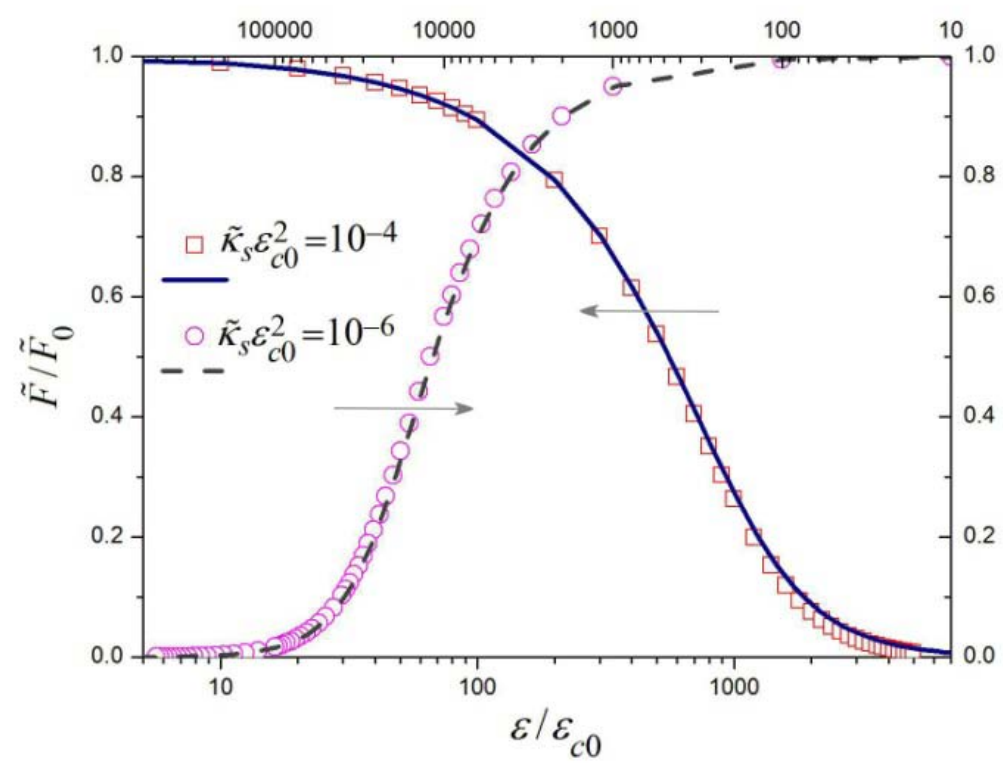


Figure A1. Filament force as a function of the nominal strain. The lines represent predictions by (12) whereas symbols correspond to exact results obtained from (A22). 